\title{
Experience with HPV self-sampling and clinician-based sampling in women attending routine cervical screening in the Netherlands
}

\author{
Nicole J. Polman ${ }^{\mathrm{a}, *}$, Yanne de Haan ${ }^{\mathrm{a}}$, Nienke J. Veldhuijzen ${ }^{\mathrm{b}}$, Daniëlle A.M. Heideman ${ }^{\mathrm{a}}$, \\ Henrica C.W. de Vet ${ }^{\mathrm{b}}$, Chris J.L.M. Meijer ${ }^{\mathrm{a}}$, Leon F.A.G. Massuger ${ }^{\mathrm{c}}$, Folkert J. van Kemenade ${ }^{\mathrm{d}}$, \\ Johannes Berkhof ${ }^{\mathrm{b}}$ \\ ${ }^{a}$ Cancer Center Amsterdam, Department of Pathology, Amsterdam UMC, location Vrije Universiteit Amsterdam, Amsterdam, the Netherlands \\ ${ }^{\mathrm{b}}$ Department of Epidemiology and Biostatistics, Amsterdam UMC, location Vrije Universiteit Amsterdam, Amsterdam, the Netherlands \\ ${ }^{\mathrm{c}}$ Department of Obstetrics and Gynaecology, Radboud University Medical Center, Nijmegen, the Netherlands \\ ${ }^{\mathrm{d}}$ Department of Pathology, Erasmus University Medical Center, Rotterdam, the Netherlands
}

\section{A R T I C L E I N F O}

\section{Keywords:}

Experience

Preference

Cervical screening

HPV testing

Self-sampling

Clinician-based sampling

\begin{abstract}
A B S T R A C T
Several countries offer HPV self-sampling for screening non-attendees. It is assumed that screening attendees also prefer self-sampling to clinician-based sampling, however, little research has been conducted with respect to this. Women participating in the IMPROVE-study were randomised (1:1) to self- or clinician-collected HPV testing, and HPV-positive women were retested using the other collection method. Three different questionnaires were sent out among a subset of participating women: Q1) HPV-positive women from both study groups were asked about their experiences with self-sampling and clinician-based sampling $(n=497)$; Q2) HPV-negative women from the self-sampling group were asked about their experiences with self-sampling $(n=2366)$; and Q3) HPV-negative women in the clinician-collection group were asked about their experiences with clinician-based sampling $(n=2092)$. Response rates ranged from 71.6 to $79.4 \%$. Women reported significantly lower levels of shame, nervousness, discomfort and pain during self-sampling compared to clinician-based sampling. However, trust in correct sampling was significantly higher during clinician-based sampling. The majority of women in group Q1 preferred self-sampling (76.5\%) to clinician-based sampling (11.9\%) in future screening, while $11.6 \%$ of women reported to have no preference for either method. To conclude, women from a regular screening population have a positive attitude towards self-sampling but express some concerns with respect to accuracy. The majority prefers self-sampling to clinician-based sampling in future screening. Based on these results, a screening approach where women can choose for either self-sampling or clinician-based sampling seems highly justifiable.
\end{abstract}

\section{Introduction}

Testing for the presence of high-risk human papillomavirus (HPV) provides better protection against cervical cancer and high-grade cervical intraepithelial neoplasia (CIN) than cytology testing (Arbyn et al., 2012; Ronco et al., 2014). Therefore, several countries have replaced cytology by HPV testing as the primary screening method (Wentzensen et al., 2017). While cytology cannot be performed on a self-collected cervicovaginal sample due to the lack of a sufficient number of intact cervical cells (indicator cells), HPV testing can be performed reliably on self-sampled specimen (HPV self-sampling) (Garcia et al., 2003). Hence, the introduction of HPV-based screening opens up the possibility of offering self-sampling to women.
Studies have shown that offering HPV self-sampling to screening non-attendees increases participation rates (Virtanen et al., 2011a; Virtanen et al., 2011b; Wikstrom et al., 2011; Giorgi Rossi et al., 2011; Szarewski et al., 2011). Self-sampling participation rates up to $34.2 \%$ were found among screening non-attendees in the Netherlands (Bais et al., 2007; Gok et al., 2010; Gok et al., 2012; Bosgraaf et al., 2015; Verhoef et al., 2014). Surveys evaluating women's experiences with self-sampling, showed that most non-attendees would prefer self-sampling to clinician-based sampling in the next screening round (Bosgraaf et al., 2015; Huynh et al., 2010; Nelson et al., 2017). Besides, a metaanalysis indicated that the sensitivity of HPV testing on self-collected and clinician-collected specimens for detection of cervical intraepithelial grade 2 or worse (CIN2+) are similar when HPV testing is

\footnotetext{
* Corresponding author.

E-mail address: n.polman@vumc.nl (N.J. Polman).
} 
conducted with a clinically validated PCR-based assay (Arbyn et al., 2014). Based on these results, several countries, including the Netherlands, have implemented HPV self-sampling for screening non-attendees (Dutch Health Council, 2016; Lew et al., 2013; Danish Health Council, 2017).

HPV self-sampling may also be considered as a primary screening instrument in the screening population as a whole. Implementation of self-sampling as a primary screening option would reduce the costs of cervical screening as it obviates the need of smear taking at the physician's office The accuracy of HPV self-sampling as compared to HPV testing on physician-collected specimens in women invited for routine screening has recently been evaluated in the IMPROVE study (Polman et al., 2019). The IMPROVE study, a randomised non-inferiority trial among 16,410 women from a routine screening population in the Netherlands, showed that HPV self-sampling has similar sensitivity and specificity as clinician-based HPV testing for detection of CIN2+ and CIN3 +, further supporting the implementation of HPV self-sampling as a primary screening option. It is generally hypothesized that screening attendees, alike most non-attendees, would prefer self-sampling to clinician-based sampling. However, little research has yet been conducted with respect to experiences and preferences of regular screening attendees.

The aim of this study was to evaluate experiences and preferences with HPV self-sampling and clinician-based sampling among women participating in routine screening. In order to evaluate this, we did a survey among a subset of women who participated in the IMPROVE study.

\section{Methods}

\subsection{Study population}

The IMPROVE study (Polman et al., 2019) was designed to assess the non-inferiority of HPV self-sampling as compared to clinician-based HPV testing. Women were invited to participate in the IMPROVE study in 2015 and 2016. At that time, routine cervical screening in the Netherlands was cytology-based and women aged 30-60 years were invited every 5 years for sample collection for cervical cytology at a general practitioner's practice. A total of 16,410 women provided informed consent to participate. These women were randomised $(1: 1)$ to the intervention group (HPV self-sampling using the Evalyn Brush, Rovers Medical Devices B.V., Oss, The Netherlands) and the control group (clinician-based HPV testing using the Cervex Brush, Rovers Medical Devices B.V., Oss, The Netherlands). Both self-sampling and clinician-based sampling were offered free of charge, as is participation in routine screening in the Netherlands. Both within the IMPROVE study and within routine screening, women were informed that possible additional research in case of a positive screening test result could entail costs. The IMPROVE study was approved by the Ministry of Public Health (no. 2014/32) and is registered in the Dutch Trial Register (NTR5078).

To assess the relative sensitivity of self-collected compared to clinician-based HPV testing in the IMPROVE study, HPV-positive women in the intervention group were also tested for HPV on a clinician-collected sample, and HPV-positive women in the control group were requested to send in a self-sample for HPV testing. This second test (i.e. cross-test) was also free of charge.

For this survey, three different questionnaires were developed (see Fig. 1): questionnaire 1 (Q1): HPV-positive women from both study groups were asked about their experiences with both self-sampling and clinician-based sampling; questionnaire 2 (Q2): HPV-negative women from the intervention group were asked about their experiences with self-sampling; and questionnaire 3 (Q3): HPV-negative women from the control group were asked about their experiences with clinician-based sampling.

Invitations to participate in the questionnaire study were sent out in
January 2017. Because of privacy and ethical reasons, questionnaires were completely anonymous and therefore could not be linked to the IMPROVE study on the individual level. To minimize the risk of recall bias, women who tested HPV positive between June and November 2016 ( $n=497$ HPV-positive women [Q1]) and women who tested HPV negative between September and November $2016(n=4458 ; 2366$ women in the intervention group [Q2] and 2092 in the control group [Q3])were invited to participate.

\subsection{Questionnaire}

The questionnaires (see supplementary material) were developed based on previous studies evaluating experiences with HPV self-sampling (Bosgraaf et al., 2015; Sultana et al., 2015; Virtanen et al., 2014; Karjalainen et al., 2016; Berner et al., 2013; Waller et al., 2006). The questionnaires were tested in an external pilot group of ten women within the screening age in order to make sure that the questions were clear and understandable.

The questionnaires contained three to five components. The first component contained questions about age, level of education and screening history and was included in Q1, Q2, and Q3. The second component contained questions about the intelligibility of the given instructions and the usability of the self-sampling device and was included in Q1 and Q2. The third component contained questions about experiences with self-sampling and/or clinician-based sampling (discomfort, pain, nervousness, shame, privacy and trust in correct execution of the sampling procedure) and was included in Q1, Q2 and Q3. In addition, Q2 and Q3 had one extra question regarding trust in accuracy of the HPV result. HPV-positive women in the IMPROVE study were advised to undergo follow-up testing and had often already received additional test results, making this question unsuitable for HPV-positives. The fourth component contained questions about preference for participation in future screening (self-sampling, clinician-based sampling, or no preference) and was included in Q1 and Q2. The fifth component contained questions about knowledge with respect to HPV and cervical cancer, feelings of concern after receiving the HPV-positive test result, and whether the provided information after receiving the HPV-positive test result was reassuring. Since we aimed to evaluate what information HPV-positive women wanted to receive and how they could best be reassured after receiving the test result, this part of the questionnaire was only included in Q1. Women could either fill in the questionnaire on paper and sent it back using the provided return envelope, or $\log$ in and fill in the questionnaire online. Formatted responses were either binary (yes/no) or on a five point Likert-type scale.

\subsection{Statistical analysis}

Differences in age between women who participated in Q2 versus Q3 were analysed with the Mann-Whitney $U$ test, while differences in level of education and time to previous cervical smear were analysed with the Chi-square test.

Scores were filled in on a five-point scale and are presented with mean and median scores. Differences in scores between self-sampling and clinician-based sampling among HPV-positive women (Q1) were analysed using the Wilcoxon Signed-Rank test, while differences in scores between HPV-negative women from the self-sampling and clinician-based sampling group (Q2 vs. Q3) were analysed using the Chisquare test. The presented $p$-values are Bonferroni corrected (multiplied by the number of hypotheses tested, i.e. multiplied by 13).

Preference in future screening was only analysed for women who had experience with both self-sampling and clinician-based sampling and therefore were able to make an informed decision regarding their preference for the next screening round. Therefore, only HPV-positive women (Q1) and HPV-negative women from the intervention group (Q2) who indicated that they had participated in cervical screening before were included in this analysis. The effect of age on preference in 


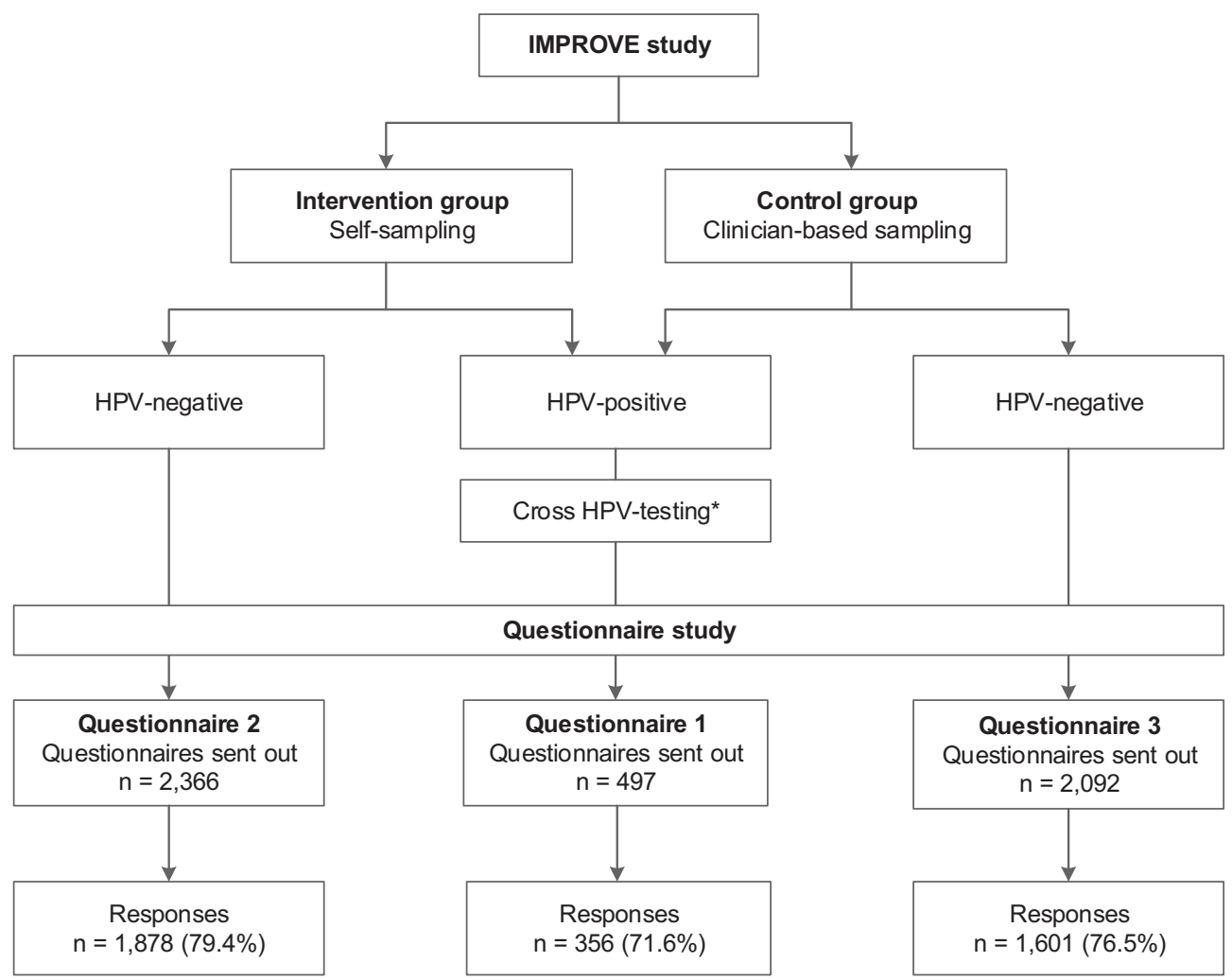

Fig. 1. Flowchart of the IMPROVE study design, including information on participation in the questionnaire study.

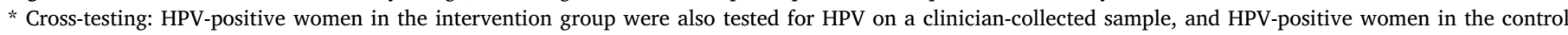
group were also tested for HPV on a self-sample.

future screening was evaluated with the Kruskal Wallis test, while differences between lower and higher educated women on preference in future screening were evaluated with the Chi-square test. As there was a small percentage of missing data, these were excluded for analysis. Data analysis was performed with IBM SPSS Statistics version 22.0 .

\section{Results}

\subsection{Response rate and study cohort characteristics}

Response rates of the Q1, Q2, and Q3 group ranged from 71.6\% to $79.4 \%$ (see Fig. 1). Overall, $68.0 \%$ of women $(n=2608)$ completed the questionnaire on paper and $32.0 \%(n=1227)$ completed the questionnaire online. Demographic characteristics of the questionnaire responders are shown in Table 1 . There were no differences with respect to age, educational level, and time to previous cervical smear between women filling out Q2 and Q3 $(p=0.886, p=0.916$, and $p=0.083$, respectively).

\subsection{Self-sampling instructions and usability}

Results regarding intelligibility of the self-sampling instructions and usability of the self-sampling device, both for HPV-positive women in the Q1 group and HPV-negative women in the self-sampling group Q2, are shown in Table 2. Overall, $>95 \%$ of women reported a very to extremely good intelligibility of the self-sampling instructions $(95.5 \%$ in Q1 and 96.5\% in Q3). Moreover, 77.7\% of women in Q1 group and $84.6 \%$ of women in Q3 group reported a very to extremely good usability of the self-sampling device.

\subsection{Experience of self-sampling versus clinician-based sampling}

Results among HPV-positive women (Q1) who performed both self- sampling and clinician-based sampling are shown in Table 3. Women reported significantly less discomfort during self-sampling as compared to clinician-based sampling: $68.7 \%$ reported no discomfort at all during self-sampling, compared to $19.2 \%$ during clinician-based sampling $(p=0.002)$. Additionally, $80.8 \%$ of women reported no pain at all during self-sampling, compared to $33.0 \%$ of women during clinicianbased sampling $(p<0.001)$. Women reported significantly lower levels of nervousness and shame during self-sampling than during clinician-based sampling: $62.0 \%$ vs. $27.6 \%$ reported no nervousness at all $(p=0.018)$ and $92.1 \%$ vs. $37.3 \%$ reported no shame at all $(p<0.001)$. The vast majority of women experienced a high degree of privacy during self-sampling (93.2\%), while only $31.4 \%$ reported this during clinician-based sampling $(p<0.001)$. Finally, $53.7 \%$ of women reported maximal trust in correct execution of the self-sampling procedure, compared to $73.3 \%$ that reported extreme trust in correct execution of the clinician-based sampling procedure $(p<0.001)$.

Results of HPV-negative women from the self-sampling group versus HPV-negative women from the clinician-based sampling group (Q2 vs. Q3) are shown in Table 4. The majority (76.9\%) of women from the self-sampling group reported no discomfort at all while performing selfsampling, while only $28.3 \%$ of women from the control group reported no discomfort during clinician-based sampling $(p<0.001)$. Moreover, $81.9 \%$ of women in the self-sampling group reported no pain, compared to $40.1 \%$ of women in the clinician-based sampling group ( $<<0.001$ ). Women from the self-sampling group reported significantly lower levels of nervousness and shame as compared to women from the clinicianbased sampling group: $63.7 \%$ vs. $43.0 \%$ reported no nervousness at all ( $\mathrm{p}<0.001$ ) and $91.4 \%$ vs. $47.9 \%$ reported no shame at all ( $\mathrm{p}<0.001$ ). With regard to privacy, $95.5 \%$ of women in the selfsampling group reported extremely high privacy compared to $61.8 \%$ of women in the clinician-based sampling group ( $p<0.001)$. The percentage of women who reported extreme trust in correct execution of the sampling procedure was $53.3 \%$ in the self-sampling group and 
Table 1

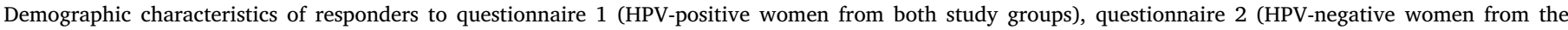
intervention group), and questionnaire 3 (HPV-negative women from the control group).

\begin{tabular}{|c|c|c|c|c|c|c|}
\hline \multirow[b]{2}{*}{ Characteristics } & \multicolumn{2}{|c|}{ Questionnaire 1} & \multicolumn{2}{|c|}{ Questionnaire 2} & \multicolumn{2}{|c|}{ Questionnaire 3} \\
\hline & $\mathrm{n}$ & $\%$ & $\mathrm{n}$ & $\%$ & $\mathrm{n}$ & $\%$ \\
\hline Mean age in years (range) & 43.7 & $(30-60)$ & 48.4 & $(30-63)$ & 48.4 & $(30-61)$ \\
\hline \multicolumn{7}{|l|}{ Age cohorts } \\
\hline $30-33$ & 74 & $20.9 \%$ & 105 & $5.6 \%$ & 82 & $5.1 \%$ \\
\hline $34-38$ & 46 & $13.0 \%$ & 129 & $6.9 \%$ & 130 & $8.1 \%$ \\
\hline $39-43$ & 35 & $9.9 \%$ & 246 & $13.2 \%$ & 207 & $13.0 \%$ \\
\hline $44-48$ & 51 & $14.4 \%$ & 361 & $19.3 \%$ & 266 & $16.7 \%$ \\
\hline $49-53$ & 67 & $18.9 \%$ & 316 & $16.9 \%$ & 295 & $18.5 \%$ \\
\hline $54-58$ & 53 & $15.0 \%$ & 376 & $20.1 \%$ & 344 & $21.5 \%$ \\
\hline $59-63$ & 28 & $7.9 \%$ & 337 & $18.0 \%$ & 273 & $17.1 \%$ \\
\hline Missing & 2 & - & 8 & - & 4 & - \\
\hline \multicolumn{7}{|l|}{ Level of education } \\
\hline No or primary education & 1 & $0.3 \%$ & 13 & $0.7 \%$ & 12 & $0.8 \%$ \\
\hline Secondary education & 48 & $13.6 \%$ & 396 & $21.1 \%$ & 321 & $20.2 \%$ \\
\hline Intermediate/Higher vocational education & 212 & $59.9 \%$ & 1116 & $59.6 \%$ & 951 & $59.7 \%$ \\
\hline University & 93 & $26.3 \%$ & 348 & $18.6 \%$ & 309 & $19.4 \%$ \\
\hline Missing & 2 & - & 5 & - & 8 & - \\
\hline \multicolumn{7}{|l|}{ Time to previous cervical smear } \\
\hline Women aged $<34$ years (first invitation for cervical screening) & 74 & $21.1 \%$ & 104 & $5.6 \%$ & 82 & $5.2 \%$ \\
\hline \multicolumn{7}{|l|}{ Women aged $\geq 34$ years } \\
\hline$<7$ years & 243 & $69.2 \%$ & 1545 & $83.7 \%$ & 1353 & $86.6 \%$ \\
\hline $7-12$ years & 16 & $4.6 \%$ & 107 & $5.8 \%$ & 79 & $5.1 \%$ \\
\hline$\geq 13$ years ago & 6 & $1.7 \%$ & 22 & $1.2 \%$ & 11 & $0.7 \%$ \\
\hline Never & 12 & $3.4 \%$ & 68 & $3.7 \%$ & 38 & $2.4 \%$ \\
\hline Missing & 5 & - & 32 & - & 38 & - \\
\hline Total & 356 & & 1878 & & 1601 & \\
\hline
\end{tabular}

$64.4 \%$ in the clinician-based sampling group ( $\mathrm{p}<0.001$ ). Finally, $54.1 \%$ of women in the self-sampling group reported extreme trust towards the test result, as compared to $59.4 \%$ of women in the clinician-based sampling group $(p=0.002)$.

\subsection{Preference in future screening}

Among 356 HPV-positive women who participated in both selfsampling and clinician-based sampling within the IMPROVE trial (Q1), 353 filled in the question about a preference for future screening. The vast majority of these women $(76.5 \%)$ reported to prefer self-sampling in future screening, $11.9 \%$ reported to prefer clinician-based sampling and $11.6 \%$ reported to have no preference for either method. The preferred screening method in future screening was not influenced by age, level of education, or screening history $(p=0.502, p=0.811$, and $p=0.550$, respectively).

A total of 1674 (89.1\%) of 1878 HPV-negative women in the selfsampling group (Q2) reported that they had previously participated in screening. Of these, 1662 filled in the question about preference in future screening. The vast majority of these women $(89.4 \% ; n=1486)$ reported to prefer self-sampling to clinician-based sampling in future screening. In contrast, $2.8 \%(n=47)$ of women reported to prefer clinician-based sampling for future screening and $7.8 \%(n=129)$ of women reported that they had no preference for either method. The preferred screening method in future screening was not influenced by age, level of education, or screening history $(p=0.894, p=0.086$, and $p=0.434$, respectively). When we analysed preference for future screening among all HPV-negative women (Q2 and Q3), irrespective of whether they have experience with both sampling methods, 2420 (70.1\%) reported to prefer self-sampling to clinician-based sampling in future screening, while 439 (12.7\%) reported to prefer clinician-based sampling and $595(17.2 \%)$ reported to have no preference.

\subsection{Knowledge and attitudes}

A total of 157 (44.5\%) of 353 HPV-positive women reported to be fully familiar with the relation between HPV and cervical (pre)cancer. However, also a substantial proportion of women $(28.3 \%, 100$ of 353) reported not to be familiar at all with this causal relationship (mean score: 3.4). Among HPV-positive women, $17.0 \%$ (60 of 353) reported not to be concerned or only slightly concerned, after being informed about the HPV-positive test result, while $51.8 \%$ of women reported to be very or extremely concerned. Most women (49.7\%; 175 of 352) reported that the information provided in the result letter, via the website and/or via the information line was very to extremely reassuring, while $15.9 \%$ (56 of 352) reported that the provided

Table 2

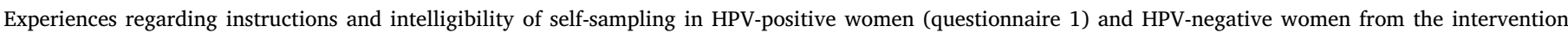
group (questionnaire 2).

\begin{tabular}{|c|c|c|c|c|c|c|c|c|c|c|c|c|c|c|c|c|}
\hline & \multirow[b]{2}{*}{ Mean } & \multirow[b]{2}{*}{$(95 \% \mathrm{CI})$} & \multirow[b]{2}{*}{ Median } & \multirow[b]{2}{*}{ (Range) } & \multicolumn{2}{|c|}{ 1- not at all } & \multicolumn{2}{|c|}{ 2-slightly } & \multicolumn{2}{|c|}{ 3-moderately } & \multicolumn{2}{|c|}{ 4-very } & \multicolumn{2}{|c|}{ 5-extremely } & \multirow{2}{*}{$\frac{\text { Total }}{\mathrm{n}}$} & Missing \\
\hline & & & & & $\mathrm{n}$ & $\%$ & $\mathrm{n}$ & $\%$ & $\mathrm{n}$ & $\%$ & $\mathrm{n}$ & $\%$ & $\mathrm{n}$ & $\%$ & & $\mathrm{n}$ \\
\hline \multicolumn{17}{|c|}{ Intelligibility of self-sampling instructions } \\
\hline Questionnaire 1 & 4.7 & $(4.61-4.73)$ & 5 & $(2-5)$ & 0 & $0.0 \%$ & 2 & $0.6 \%$ & 14 & $3.9 \%$ & 83 & $23.3 \%$ & 257 & $72.2 \%$ & 356 & 0 \\
\hline Questionnaire 2 & 4.7 & $(4.67-4.72)$ & 5 & $(1-5)$ & 4 & $0.2 \%$ & 14 & $0.7 \%$ & 47 & $2.5 \%$ & 413 & $22.0 \%$ & 1397 & $74.5 \%$ & 1875 & 3 \\
\hline \multicolumn{17}{|c|}{ Usability of self-sampling device } \\
\hline Questionnaire 1 & 3.1 & $(2.99-3.27)$ & 5 & $(1-5)$ & 28 & $7.9 \%$ & 38 & $10.7 \%$ & 13 & $3.7 \%$ & 55 & $15.5 \%$ & 220 & $62.1 \%$ & 354 & 2 \\
\hline Questionnaire 2 & 3.4 & $(3.32-3.42)$ & 5 & $(1-5)$ & 92 & $4.9 \%$ & 131 & $7.0 \%$ & 66 & $3.5 \%$ & 287 & $15.3 \%$ & 1296 & $69.2 \%$ & 1872 & 6 \\
\hline
\end{tabular}


Table 3

Experiences with self-sampling and clinician-based sampling (Q1).

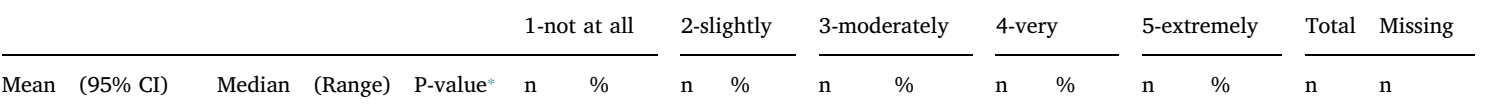

\begin{tabular}{|c|c|c|c|c|c|c|c|c|c|c|c|c|c|c|c|c|c|}
\hline \multicolumn{18}{|l|}{ Discomfort } \\
\hline Self-sampling & 1.4 & $(1.35-1.49)$ & 1 & $(1-5)$ & \multirow[t]{2}{*}{$<0.001$} & 244 & $68.7 \%$ & 84 & $23.7 \%$ & 19 & $5.4 \%$ & 6 & $1.7 \%$ & 2 & $0.6 \%$ & 355 & 1 \\
\hline Clinician-based sampling & 2.8 & $(2.70-2.96)$ & 3 & $(1-5)$ & & 68 & $19.2 \%$ & 71 & $20.0 \%$ & 103 & $29.0 \%$ & 80 & $22.5 \%$ & 33 & $9.3 \%$ & 355 & 1 \\
\hline \multicolumn{18}{|l|}{ Pain } \\
\hline Self-sampling & 1.3 & $(1.22-1.36)$ & 1 & $(1-5)$ & \multirow[t]{2}{*}{$<0.001$} & 286 & $80.8 \%$ & 45 & $12.7 \%$ & 14 & $4.0 \%$ & 6 & $1.7 \%$ & 3 & $0.8 \%$ & 354 & 2 \\
\hline Clinician-based sampling & 2.4 & $(2.25-2.51)$ & 2 & $(1-5)$ & & 116 & $33.0 \%$ & 86 & $24.4 \%$ & 72 & $20.5 \%$ & 57 & $16.2 \%$ & 21 & $6.0 \%$ & 352 & 4 \\
\hline \multicolumn{18}{|l|}{ Nervousness } \\
\hline Self-sampling & 1.6 & $(1.46-1.64)$ & 1 & $(1-5)$ & \multirow[t]{2}{*}{$<0.001$} & 220 & $62.0 \%$ & 95 & $26.8 \%$ & 24 & $6.8 \%$ & 13 & $3.7 \%$ & 3 & $0.8 \%$ & 355 & 1 \\
\hline Clinician-based sampling & 2.6 & $(2.50-2.77)$ & 3 & $(1-5)$ & & 98 & $27.6 \%$ & 68 & $19.2 \%$ & 84 & $23.7 \%$ & 73 & $20.6 \%$ & 32 & $9.0 \%$ & 355 & 1 \\
\hline \multicolumn{18}{|l|}{ Shame } \\
\hline Self-sampling & 1.1 & $(1.05-1.13)$ & 1 & $(1-4)$ & \multirow[t]{2}{*}{$<0.001$} & 327 & $92.1 \%$ & 24 & $6.8 \%$ & 3 & $0.8 \%$ & 1 & $0.3 \%$ & 0 & $0.0 \%$ & 355 & 1 \\
\hline Clinician-based sampling & 2.2 & $(2.09-2.35)$ & 2 & $(1-5)$ & & 132 & $37.3 \%$ & 88 & $24.9 \%$ & 76 & $21.5 \%$ & 41 & $11.6 \%$ & 17 & $4.8 \%$ & 354 & 2 \\
\hline \multicolumn{18}{|l|}{ Privacy } \\
\hline Self-sampling & 4.8 & $(4.71-4.89)$ & 5 & $(1-5)$ & \multirow[t]{2}{*}{$<0.001$} & 15 & $4.2 \%$ & 1 & $0.3 \%$ & 1 & $0.3 \%$ & 7 & $2.0 \%$ & 329 & $93.2 \%$ & 353 & 3 \\
\hline Clinician-based sampling & 3.6 & $(3.44-3.71)$ & 4 & $(1-5)$ & & 31 & $8.8 \%$ & 50 & $14.1 \%$ & 65 & $18.4 \%$ & 97 & $27.4 \%$ & 111 & $31.4 \%$ & 354 & 2 \\
\hline \multicolumn{18}{|c|}{ Trust in correct execution of sampling procedure } \\
\hline Self-sampling & 4.3 & $(4.25-4.43)$ & 5 & $(1-5)$ & \multirow[t]{2}{*}{$<0.001$} & 6 & $1.7 \%$ & 12 & $3.4 \%$ & 28 & $7.9 \%$ & 119 & $33.4 \%$ & 191 & $53.7 \%$ & 356 & 0 \\
\hline Clinician-based sampling & 4.7 & $(4.61-4.73)$ & 5 & $(1-5)$ & & 1 & $0.3 \%$ & 3 & $0.8 \%$ & 13 & $3.7 \%$ & 78 & $21.9 \%$ & 261 & $73.3 \%$ & 356 & 0 \\
\hline
\end{tabular}

* Bonferroni corrected p-value.

information was not or only slightly reassuring.

\section{Discussion}

We aimed to evaluate experiences with HPV self-sampling as compared to clinician-based sampling among women from a routine Dutch screening cohort. Overall, results show that experiences with selfsampling were rated more positive than experiences with clinicianbased sampling. Intelligibility of the self-sampling instructions was rated good and usability of the self-sampling device was rated moderately good. Women experienced significantly lower levels of discomfort, pain, nervousness and shame with self-sampling, and significantly higher levels of privacy. Trust in correct execution of sampling method and trust in accuracy of the HPV result were slightly lower with self-sampling as compared to clinician-based sampling. Nonetheless, the majority of women reported to prefer self-sampling in future screening.

Previous studies among non-attendees who were offered self-sampling showed that women found self-sampling to be more convenient, less embarrassing, less uncomfortable and less painful as compared to clinician-based sampling (Sultana et al., 2015). In addition, women indicated that they would prefer self-sampling to clinician-based

Table 4

Experiences with self-sampling in the intervention group (Q2) versus experiences with clinician-based sampling in the control group (Q3).

\begin{tabular}{|c|c|c|c|c|c|c|c|c|c|c|c|c|c|c|c|c|c|}
\hline & & & & & & \multicolumn{2}{|c|}{ 1-not at all } & \multicolumn{2}{|c|}{ 2-slightly } & \multicolumn{2}{|c|}{ 3-moderately } & \multicolumn{2}{|c|}{ 4-very } & \multicolumn{2}{|c|}{ 5-extremely } & \multirow{2}{*}{$\frac{\text { Total }}{\mathrm{n}}$} & Missing \\
\hline & Mean & $(95 \% \mathrm{CI})$ & Median & (Range) & P-value* & $\mathrm{n}$ & $\%$ & $\mathrm{n}$ & $\%$ & $\mathrm{n}$ & $\%$ & $\mathrm{n}$ & $\%$ & $\mathrm{n}$ & $\%$ & & $\mathrm{n}$ \\
\hline \multicolumn{18}{|l|}{ Discomfort } \\
\hline Self-sampling & 1.3 & $(1.31-1.37)$ & 1 & $(1-5)$ & $<0.001$ & 1441 & $76.9 \%$ & 285 & $15.2 \%$ & 96 & $5.1 \%$ & 42 & $2.2 \%$ & 10 & $0.5 \%$ & 1874 & 4 \\
\hline $\begin{array}{l}\text { Clinician-based } \\
\text { sampling }\end{array}$ & 2.4 & $(2.34-2.46)$ & 2 & $(1-5)$ & & 451 & $28.3 \%$ & 474 & $29.7 \%$ & 355 & $22.3 \%$ & 218 & $13.7 \%$ & 97 & $6.1 \%$ & 1595 & 6 \\
\hline \multicolumn{18}{|l|}{ Pain } \\
\hline Self-sampling & 1.3 & (1.25-1.59) & 1 & $(1-5)$ & $<0.001$ & 1534 & $81.9 \%$ & 224 & $12.0 \%$ & 58 & $3.1 \%$ & 44 & $2.3 \%$ & 13 & $0.7 \%$ & 1873 & 5 \\
\hline $\begin{array}{l}\text { Clinician-based } \\
\text { sampling }\end{array}$ & 2.1 & $(2.08-2.20)$ & 2 & $(1-5)$ & & 639 & $40.1 \%$ & 427 & $26.8 \%$ & 264 & $16.6 \%$ & 190 & $11.9 \%$ & 75 & $4.7 \%$ & 1595 & 6 \\
\hline \multicolumn{18}{|l|}{ Nervousness } \\
\hline Self-sampling & 1.6 & $(1.52-1.61)$ & 1 & $(1-5)$ & $<0.001$ & 1194 & $63.7 \%$ & 401 & $21.4 \%$ & 182 & $9.7 \%$ & 80 & $4.3 \%$ & 16 & $0.9 \%$ & 1873 & 5 \\
\hline $\begin{array}{l}\text { Clinician-based } \\
\text { sampling }\end{array}$ & 2.1 & $(2.00-2.10)$ & 2 & $(1-5)$ & & 687 & $43.0 \%$ & 403 & $25.2 \%$ & 304 & $19.0 \%$ & 154 & $9.6 \%$ & 49 & $3.1 \%$ & 1597 & 4 \\
\hline \multicolumn{18}{|l|}{ Shame } \\
\hline Self-sampling & 1.1 & (1.09-1.13) & 1 & $(1-5)$ & $<0.001$ & 1712 & $91.4 \%$ & 126 & $6.7 \%$ & 23 & $1.2 \%$ & 6 & $0.3 \%$ & 6 & $0.3 \%$ & 1873 & 5 \\
\hline $\begin{array}{l}\text { Clinician-based } \\
\text { sampling }\end{array}$ & 1.9 & (1.87-1.97) & 2 & $(1-5)$ & & 764 & $47.9 \%$ & 414 & $26.0 \%$ & 233 & $14.6 \%$ & 145 & $9.1 \%$ & 39 & $2.4 \%$ & 1595 & 6 \\
\hline \multicolumn{18}{|l|}{ Privacy } \\
\hline Self-sampling & 4.9 & $(4.84-4.90)$ & 5 & $(1-5)$ & $<0.001$ & 51 & $2.7 \%$ & 3 & $0.2 \%$ & 7 & $0.4 \%$ & 24 & $1.3 \%$ & 1788 & $95.5 \%$ & 1873 & 5 \\
\hline $\begin{array}{l}\text { Clinician-based } \\
\text { sampling }\end{array}$ & 4.3 & $(4.29-4.39)$ & 5 & $(1-5)$ & & 67 & $4.2 \%$ & 58 & $3.6 \%$ & 130 & $8.1 \%$ & 354 & $22.2 \%$ & 987 & $61.8 \%$ & 1596 & 5 \\
\hline \multicolumn{18}{|c|}{ Trust in correct execution of sampling procedure } \\
\hline Self-sampling & 4.4 & $(4.33-4.41)$ & 5 & $(1-5)$ & $<0.001$ & 7 & $0.4 \%$ & 42 & $2.2 \%$ & 194 & $10.4 \%$ & 631 & $33.7 \%$ & 998 & $53.3 \%$ & 1872 & 6 \\
\hline $\begin{array}{l}\text { Clinician-based } \\
\text { sampling }\end{array}$ & 4.5 & $(4.48-4.56)$ & 5 & $(1-5)$ & & 13 & $0.8 \%$ & 29 & $1.8 \%$ & 107 & $6.7 \%$ & 420 & $26.3 \%$ & 1030 & $64.4 \%$ & 1599 & 2 \\
\hline \multicolumn{18}{|l|}{ Trust in test result } \\
\hline Self-sampling & 4.4 & (4.41-4.47) & 5 & $(1-5)$ & 0.005 & 4 & $0.2 \%$ & 11 & $0.6 \%$ & 157 & $8.4 \%$ & 687 & $36.7 \%$ & 1011 & $54.1 \%$ & 1870 & 8 \\
\hline $\begin{array}{l}\text { Clinician-based } \\
\text { sampling }\end{array}$ & 4.5 & (4.47-4.53) & 5 & $(1-5)$ & & 10 & $0.6 \%$ & 12 & $0.8 \%$ & 88 & $5.5 \%$ & 538 & $33.7 \%$ & 947 & $59.4 \%$ & 1595 & 6 \\
\hline
\end{tabular}

* Bonferroni corrected p-value. 
sampling for future screening (Bosgraaf et al., 2015; Sultana et al., 2015; Virtanen et al., 2014; Karjalainen et al., 2016). Results of the current study are in line with previous studies and show that experiences and preferences regarding self-sampling do also apply to a routine screening population. Previous studies that evaluated self-sampling experiences in a routine screening population showed, as in the current study, that women reported self-sampling to be convenient and userfriendly (Waller et al., 2006; Ketelaars et al., 2017). Moreover, 63\% of women reported to prefer self-sampling over clinician-based sampling for future screening, as compared to about $80 \%$ in the current study (Ketelaars et al., 2017).

As in several previous studies (Sultana et al., 2015; Waller et al., 2006), women in the current study performing self-sampling reported significantly lower levels of trust in correct execution of the sampling procedure and in the test result as compared to clinician-based sampling. Trust in correct execution of sampling procedure and in the test result are likely to increase when additional evidence about the clinical accuracy of HPV self-sampling in a routine screening population becomes available to women. In this regard, the results of the IMPROVE study, showing that HPV self-sampling is non-inferior to clinician-based HPV testing with respect to detection of CIN2 + and CIN3 +, are encouraging and may help to reduce uncertainties about HPV self-sampling. If women become informed that self-sampling is a reliable and safe method for participation in cervical screening, it is to be expected that an even higher proportion of women would prefer self-sampling. Here, the authorities responsible for screening programs, as well as general practitioners, physician assistants and gynaecologists, play an important role in informing and educating women, and are likely to have major impact on the acceptability of HPV self-sampling.

A majority of HPV-negative women (89\%) indicated to prefer selfsampling to clinician-based sampling in future screening. HPV-positives also reported a preference towards self-sampling, however, slightly less pronounced: $77 \%$ preferred self-sampling. A possible explanation for the difference in preference between HPV-negative and HPV-positive women is that self-sampling HPV-positives in the IMPROVE study still had to undergo clinician-based sampling for cytology triage (Polman et al., 2019). This second step may be experienced as burdensome and may have decreased HPV-positive women's preference regarding selfsampling as compared to HPV-negative women. Direct triage tests for HPV-positive samples that are applicable on self-collected samples may overcome this problem. Cytology testing cannot be accurately performed on self-collected samples, but molecular triage testing may provide a solution in the future (Luttmer et al., 2016; Ebisch et al., 2016; Verlaat et al., 2018; Snoek et al., 2019).

In this study, all women performing self-sampling used the Evalyn brush. Consequently, results with regard to usability, discomfort, pain, nervousness, shame, privacy, trust in correct execution of sampling procedure, and trust in accuracy of the HPV result were reported specifically for this brush-based self-sampling device. However, previous studies reported similar acceptability and experiences between brushand lavage-based self-sampling devices (Bosgraaf et al., 2015; Karjalainen et al., 2016)

Important strengths of this study are its large sample size and its setting within the nationwide screening programme in the Netherlands. However, there are also some limitations. The IMPROVE study was an opt-in study with a fairly low response rate: only 16,000 out of 185,000 invited women participated in the IMPROVE study. Moreover, among participating women, there was a higher percentage of withdrawal among women randomised to clinician-based sampling as compared to self-sampling, which may indicate a pre-existing preference for selfsampling among study participants. This may raise questions about the representativeness of the IMPROVE study for a routine screening setting. However, women in the IMPROVE cohort had a similar screening history and $\mathrm{CIN} 2+$ rate as women participating in the national screening programme, indicating that the IMPROVE study population does not represent a subset of underscreened women. The IMPROVE population may still be a cohort in which the attitude towards HPV selfsampling is more positive than in the routine screening population, although results observed in our study are consistent with other preference studies. Another limitation of this study is that women received the questionnaire a few months after participation in the IMPROVE trial. Therefore, results may be subject to recall bias. However, in order to minimize this type of bias, questionnaires were only sent to women who had participated in the IMPROVE study within six months prior to the start of the questionnaire study. Another limitation is that, because of privacy and ethical regulations in the Netherlands, questionnaires were completely anonymous and therefore could not be linked to the IMPROVE study on the individual level.

Future research should focus on the clinical accuracy of other selfsampling devices in combination with HPV assays, and on the experiences and preferences of women, and the participation rate of selfsampling as a primary screening method in routine screening. Depending on results of these studies self-sampling might be offered as a primary method in the future. Arguments that are expected to play a role in the discussion about how to screen women in the future are costeffectiveness, maximisation of screening coverage, and women their preferences.

In conclusion, results of this study show that women from a routine screening population have a positive attitude towards self-sampling but do express some concerns with respect to accuracy. Moreover, the majority of these women prefer self-sampling to clinician-based sampling in future screening. These results are supportive of self-sampling as a female-friendly primary screening method. Given that there are some concerns about the reliability of self-sampling and a minority of women prefer clinician-based sampling, a screening approach where women can choose for either method seems highly justifiable.

\section{Funding}

This work was supported by the 7th framework programme of the European Community (COHEAHR) [grant no 603019]; and the Dutch Ministry of Health, Welfare, and Sport [grant no 325973].

\section{Conflict of interest}

NJP, YH, NJV, HCWV, LFAGM and FJK have no conflict of interest.

DAMH has minority stake in Self-screen B.V., a spin-off company of Amsterdam UMC (formerly known as VU University Medical Center Amsterdam), which owns patents related to this work. DAMH has been on the speaker's bureau of Qiagen, serves occasionally on the scientific advisory boards of Pfizer and Bristol-Meyer Squibb.

CJLMM served occasionally on the scientific advisory board (expert meeting) of Qiagen and SPMSD/Merck, and served by occasion as a consultant for Qiagen. He also served occasionally on the scientific board of GSK, and has received speakers' fee from SPMSD/Merck. CJLMM has been co-investigator on a Sanofi Pasteur MSD sponsored trial, of which his institute received research funding. CJLMM is minority shareholder and part-time CEO of Self-Screen B.V., which holds patents on hrHPV test and methylation marker tests in cervical screening. CJLMM has very small number of shares of Qiagen.

$\mathrm{JB}$ received fees for attending expert meetings organized by Merck and GSK and travel support by DDL. Fees were collected by JB's employer.

\section{Appendix A. Supplementary data}

Supplementary data to this article can be found online at https:// doi.org/10.1016/j.ypmed.2019.04.025.

\section{References}

Arbyn, M., Ronco, G., Anttila, A., Meijer, C.J., Poljak, M., Ogilvie, G., et al., 2012. 
Evidence regarding human papillomavirus testing in secondary prevention of cervical cancer. Vaccine 30 (Suppl. 5), F88-F99.

Arbyn, M., Verdoodt, F., Snijders, P.J., Verhoef, V.M., Suonio, E., Dillner, L., et al., 2014. Accuracy of human papillomavirus testing on self-collected versus clinician-collected samples: a meta-analysis. Lancet Oncol. 15 (2), 172-183.

Bais, A.G., van Kemenade, F.J., Berkhof, J., Verheijen, R.H., Snijders, P.J., Voorhorst, F., et al., 2007. Human papillomavirus testing on self-sampled cervicovaginal brushes: an effective alternative to protect nonresponders in cervical screening programs. Int. J. Cancer 120 (7), 1505-1510.

Berner, A., Hassel, S.B., Tebeu, P.M., Untiet, S., Kengne-Fosso, G., Navarria, I., et al, 2013. Human papillomavirus self-sampling in Cameroon: women's uncertainties over the reliability of the method are barriers to acceptance. J. Low. Genit. Tract Dis. 17 (3), 235-241.

Bosgraaf, R.P., Verhoef, V.M., Massuger, L.F., Siebers, A.G., Bulten, J., de Kuyper-de Ridder, G.M., et al., 2015. Comparative performance of novel self-sampling methods in detecting high-risk human papillomavirus in 30,130 women not attending cervical screening. Int. J. Cancer 136 (3), 646-655.

Danish Health Council, 2017. Screening for cervical cancer - recomendations (In Danish) Available from: https://prodstoragehoeringspo.blob.core.windows.net/fdaf8cf3e83f-4c62-87ea-611ade1dd743/SST\%20Anbefalinger\%20livmoderhalskr\%C3\% A6ftscreening\%20-\%20H\%C3\%B8ring\%20nov\%202017.pdf.

Dutch Health Council, 2016. Law on population-based screening: renewal of national population-based screening programme for cervical cancer (in Dutch) [Available from. https://www.gezondheidsraad.nl/sites/default/files/201608_wbo_ vernieuwing_bmhk.pdf.

Ebisch, R.M., Siebers, A.G., Bosgraaf, R.P., Massuger, L.F., Bekkers, R.L., Melchers, W.J., 2016. Triage of high-risk HPV positive women in cervical cancer screening. Expert. Rev. Anticancer. Ther. 16 (10), 1073-1085.

Garcia, F., Barker, B., Santos, C., Brown, E.M., Nuno, T., Giuliano, A., et al., 2003. Crosssectional study of patient- and physician-collected cervical cytology and human papillomavirus. Obstet. Gynecol. 102 (2), 266-272.

Giorgi Rossi, P., Marsili, L.M., Camilloni, L., Iossa, A., Lattanzi, A., Sani, C., et al., 2011. The effect of self-sampled HPV testing on participation to cervical cancer screening in Italy: a randomised controlled trial (ISRCTN96071600). Br. J. Cancer 104 (2), $248-254$.

Gok, M., Heideman, D.A., van Kemenade, F.J., Berkhof, J., Rozendaal, L., Spruyt, J.W., et al., 2010. HPV testing on self-collected cervicovaginal lavage specimens as screening method for women who do not attend cervical screening: cohort study. BMJ 340, c1040.

Gok, M., van Kemenade, F.J., Heideman, D.A., Berkhof, J., Rozendaal, L., Spruyt, J.W., et al., 2012. Experience with high-risk human papillomavirus testing on vaginal brush-based self-samples of non-attendees of the cervical screening program. Int. J. Cancer 130 (5), 1128-1135.

Huynh, J., Howard, M., Lytwyn, A., 2010. Self-collection for vaginal human papillomavirus testing: systematic review of studies asking women their perceptions. J. Low. Genit. Tract Dis. 14 (4), 356-362.

Karjalainen, L., Anttila, A., Nieminen, P., Luostarinen, T., Virtanen, A., 2016. Self-sampling in cervical cancer screening: comparison of a brush-based and a lavage-based cervicovaginal self-sampling device. BMC Cancer 16, 221.

Ketelaars, P.J.W., Bosgraaf, R.P., Siebers, A.G., Massuger, L., van der Linden, J.C., Wauters, C.A.P., et al., 2017. High-risk human papillomavirus detection in selfsampling compared to physician-taken smear in a responder population of the Dutch cervical screening: results of the VERA study. Prev. Med. 101, 96-101.

Lew, J.B.S.K., Smith, M., et al., 2013. National Cervical Screening Program Renewal: Effectiveness Modelling and Economics Evaluation in the Australian Setting. Medical
Services Advisory Committee Australia MSAC Application No. 1276 [Available from: http://www.cancerscreening.gov.au/internet/screening/publishing.nsf/Content/ E6A211A6FFC29E2CCA257CED007FB678/\$File/Executive\%20Summary $\% 20$ notated\%2013.6.14.pdf.

Luttmer, R., De Strooper, L.M., Steenbergen, R.D., Berkhof, J., Snijders, P.J., Heideman, D.A., et al., 2016. Management of high-risk HPV-positive women for detection of cervical (pre)cancer. Expert. Rev. Mol. Diagn. 16 (9), 961-974.

Nelson, E.J., Maynard, B.R., Loux, T., Fatla, J., Gordon, R., Arnold, L.D., 2017. The acceptability of self-sampled screening for HPV DNA: a systematic review and metaanalysis. Sex. Transm. Infect. 93 (1), 56-61.

Polman, N.J., Ebisch, R.M., Heideman, D.A., Melchers, W.J., Bekkers, R.L., Molijn, A.C., et al., 2019. Clinical performance of HPV testing on self-collected versus cliniciancollected samples: results of a randomised paired screen-positive non-inferiority trial. Lancet Oncol. 20 (2), 229-238. https://doi.org/10.1016/S1470-2045(18)30763-0. (Epub 2019 Jan 15).

Ronco, G., Dillner, J., Elfstrom, K.M., Tunesi, S., Snijders, P.J., Arbyn, M., et al., 2014. Efficacy of HPV-based screening for prevention of invasive cervical cancer: follow-up of four European randomised controlled trials. Lancet 383 (9916), 524-532.

Snoek, B.C., Verlaat, W., Babion, I., Novianti, P.W., van de Wiel, M.A., Wilting, S.M., et al., 2019. Genome-wide microRNA analysis of HPV-positive self-samples yields novel triage markers for early detection of cervical cancer. Int. J. Cancer 144 (2), 372-379.

Sultana, F., Mullins, R., English, D.R., Simpson, J.A., Drennan, K.T., Heley, S., et al., 2015. Women's experience with home-based self-sampling for human papillomavirus testing. BMC Cancer 15, 849.

Szarewski, A., Cadman, L., Mesher, D., Austin, J., Ashdown-Barr, L., Edwards, R., et al., 2011. HPV self-sampling as an alternative strategy in non-attenders for cervical screening - a randomised controlled trial. Br. J. Cancer 104 (6), 915-920.

Verhoef, V.M., Bosgraaf, R.P., van Kemenade, F.J., Rozendaal, L., Heideman, D.A., Hesselink, A.T., et al., 2014. Triage by methylation-marker testing versus cytology in women who test HPV-positive on self-collected cervicovaginal specimens (PROHTECT-3): a randomised controlled non-inferiority trial. Lancet Oncol. 15 (3), 315-322.

Verlaat, W., Snoek, B.C., Heideman, D.A.M., Wilting, S.M., Snijders, P.J.F., Novianti, P.W., et al., 2018. Identification and validation of a 3-gene methylation classifier for HPV-based cervical screening on self-samples. Clin. Cancer Res. 24 (14), 3456-3464.

Virtanen, A., Anttila, A., Luostarinen, T., Nieminen, P., 2011a. Self-sampling versus reminder letter: effects on cervical cancer screening attendance and coverage in Finland. Int. J. Cancer 128 (11), 2681-2687.

Virtanen, A., Nieminen, P., Luostarinen, T., Anttila, A., 2011b. Self-sample HPV tests as an intervention for nonattendees of cervical cancer screening in Finland: a randomized trial. Cancer Epidemiol. Biomark. Prev. 20 (9), 1960-1969.

Virtanen, A., Nieminen, P., Niironen, M., Luostarinen, T., Anttila, A., 2014. Self-sampling experiences among non-attendees to cervical screening. Gynecol. Oncol. 135 (3), 487-494.

Waller, J., McCaffery, K., Forrest, S., Szarewski, A., Cadman, L., Austin, J., et al., 2006. Acceptability of unsupervised HPV self-sampling using written instructions. J. Med. Screen. 13 (4), 208-213.

Wentzensen, N., Arbyn, M., Berkhof, J., Bower, M., Canfell, K., Einstein, M., et al., 2017. Eurogin 2016 roadmap: how HPV knowledge is changing screening practice. Int. J. Cancer 140 (10), 2192-2200.

Wikstrom, I., Lindell, M., Sanner, K., Wilander, E., 2011. Self-sampling and HPV testing or ordinary Pap-smear in women not regularly attending screening: a randomised study. Br. J. Cancer 105 (3), 337-339. 